\section{GRP-107 IS WEAKNESS IN OLDER PATIENTS CAUSED BY INAPPROPRIATE DRUG USE?}

doi:10.1136/ejhpharm-2013-000276.107

LD Jensen, M Hallin, 0 Andersen, J Petersen. Copenhagen University Hospital Hvidovre, Clinical Research Centre, Hvidovre, Denmark

Background The use of potentially inappropriate medicines (PIMs) is common among the older population. Inappropriate drugs as well as polypharmacy expose older people to a greater risk of adverse drug reactions, and may cause hospitalizations. Only a few studies have examined the potential influence of the use of PIMs on functional status, cognitive status, quality of life, visual acuity and handgrip strength in older people.

Purpose To evaluate the relationship between the use of PIMs and weakness measured by functional status, cognitive status, quality of life, visual acuity and handgrip strength.

Materials and Methods A longitudinal study of patients aged $\geq 65$ years admitted to an Acute Medical Ward in Denmark. Data was collected from October-December 2011, at admission and at a follow-up visit 30 days after discharge. Data included information on social status, home care, functional status, cognitive status, handgrip strength, quality of life, visual acuity and medicines at time of follow-up, both over-the-counter medicines and those from the general practitioner. In addition data about days of hospitalisation, age, gender and comorbidities was also collected. PIMs were evaluated by a Danish list of PIMs, and polypharmacy was defined as a regular use of $\geq 5$ drugs. The Charlson Comorbidity Index was used to categorise comorbidities.

Results Seventy-one patients (55\% men) with a median age of 79 years participated. The median number of drugs was eight per person. Eighty percent were exposed to polypharmacy. PIMs occurred in $85 \%$ of the participants, and PIMs were associated with low function status $(\beta:-1.88, p=0.032)$, low handgrip strength $(\beta:-9.82$, $p=0.006)$ and reduced quality of life $(\beta:-0.19, p=0.0005)$, but not with morbidity as assessed by Charlson Index. Social status, home care and visual acuity were not associated with PIMs.

Conclusions PIMs are common among older people. The use of potentially inappropriate drugs has a negative impact on functional status, handgrip strength and quality of life.

No conflict of interest.

\section{GRP-108 LEAN CULTURE: AN OPPORTUNITY IN THE HOSPITAL PHARMACY PRODUCTION DEPARTMENT}

doi:10.1136/ejhpharm-2013-000276.108

1P Gillet, ${ }^{1} \mathrm{C}$ Pirlot, ${ }^{1} \mathrm{~N}$ Leonard, ${ }^{1} \mathrm{JD}$ Hecq, ${ }^{2} \mathrm{P}$ De Coster, ${ }^{3} \mathrm{~A}$ Bouzette, ${ }^{4} \mathrm{~T}$ Gnesotto. ${ }^{1} \mathrm{CHU}$ UCL Mont-Godinne, Pharmacy, Yvoir, Belgium; ${ }^{2} \mathrm{CHU}$ UCL Mont-Godinne, Executive management, Yvoir, Belgium; ${ }^{3} \mathrm{CHU}$ UCL Mont-Godinne, Operations management, Yvoir, Belgium; " ${ }^{4}$ KMqT Europe, Lean Consultancy, Yvoir, Belgium

Background Since 2009, the executive management of CHU Mont-Godinne has chosen to implement the LEAN methodology in our institution. Considering the multiple issues arising in our hospitals, a fundamental reorganisation of our processes and changing our behaviour is a matter of survival. Production accounts for about $30 \%$ of the work of the CHU Mont-Godinne hospital pharmacy. Constraints in a production facility are many: consistent quality, inventory management, delivery in time, productivity, teamwork.

Purpose To optimise resources using the LEAN tools.

Materials and Methods LEAN Tools

1. The 5S Philosophy focuses on effective workplace organisation. The objective is to achieve higher goals and thus improve the work done. There are five primary $5 \mathrm{~S}$ phases: sorting, straightening, systematic cleaning, standardising, and sustaining.
2. 'Spaghetti diagram'

Visual method

$\circ$ to depict the information flow.

$\circ$ to determine the physical flow and distance that information and people travel to process work.

3. Standardisation of practise

The process is filmed; a discussion takes place on this movie to define best practise.

This best practise is written as a standard and constitutes a training tool. The standard process is regularly revised in the context of continual improvement. Training improves the versatility of assistants in production.

4. Visual management

Improved communication through the implementation of short meetings:

What is the idea or problem? What is the action to perform? Who is responsible for it? When? Status?

Results We obtained an improvement in

- productivity: time required for preparation decreased, for example a $28 \%$ decrease for Tazocin $4 \mathrm{~g}$ diluted in glucose

- communication: two daily meetings

- standardisation of processes: 20 to $60 \%$

- versatility of assistants in production: 10 to $40 \%$

Conclusions Teamwork and standardisation of processes are now the keys elements to coping with the constraints of a production department of a hospital pharmacy and to obtaining continual quality improvement and optimising resources.

No conflict of interest.

\section{GRP-109 LENALIDOMIDE: HAEMATOLOGICAL SAFETY PROFILE}

doi:10.1136/ejhpharm-2013-000276.109

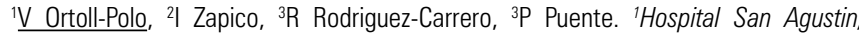
Pharmacy Service, Aviles, Spain; ${ }^{2 H o s p i t a l ~ C e n t r a l ~ d e ~ A s t u r i a s, ~ P h a r m a c y ~ S e r v i c e, ~}$ Oviedo, Spain, ${ }^{3}$ Hospital San Agustín, Pharmacy Service, Avilés, Spain

Background Lenalidomide was authorised in 2007 by EMA for the treatment of multiple myeloma (MM). It is also used off-label for myelodysplastic syndrome (MS). The drug is given orally at $25 \mathrm{mg}$ on days 1:21 (28-day therapeutic cycle) associated with dexamethasone. Dose modifications or cessation of treatment may be necessary in the event of haematological adverse events (HAEs).

Purpose To evaluate lenalidomide dose modifications in MM and MS patients due to haematological toxicity, as recommended in the EMA's drug specifications.

Materials and Methods Retrospective observational study involving 16 patients who started treatment with lenalidomide between May 2008 and September 2010. Information was collected from the clinical and pharmacotherapeutic history. If neutropenia or thrombocytopenia arose, modifications made in treatment were analysed.

Results 16 patients were found, 14 treated for MM and 2 for MS. Male/female ratio was $8 / 8$ and median age was 68.3 years (C195\%: 63.1-73.4).

Median number of cycles per patient was 6 (2-21). Considering all cycles, 98 were studied.

Pre-cycle neutropenia and thrombocytopenia were the main dose-restricting toxicities. Platelet counts $<30 \times 10^{9} / \mathrm{L}$ were found in 9 cycles; the dose was reduced in 2 patients, spaced out in 1 and both adjustments in another patient.

Neutrophil counts $<0.5 \times 10^{9} / \mathrm{L}$ were found in 12 cycles; the dose was reduced in 4 patients and spaced out in 3 . No modifications were made in $55 \%$ and $41.6 \%$ of thrombocytopenic and neutropenic patients, respectively. No records were kept about support measures such as platelet pools or granulocyte-stimulating colony-growth factors. 
Conclusions Lenalidomide's haematological toxicity is doserelated and often made worse by the basal bone marrow damage due to the haematological disease. Despite this certainty, hardly half of the patients with platelet or neutrophil damage had their dose or schedule adjusted. At this point, the patients could benefit from hospital pharmaceutical care. Important limitations of our study were lack of data about support measures and the small number of cases.

No conflict of interest.

\section{GRP-110 LINEZOLID ADVERSE REACTIONS. A ONE YEAR OVERVIEW}

doi:10.1136/ejhpharm-2013-000276.110

J Hernández, L Serrano de Lucas, B Baza, Z Baskaran, S Sautua, A de Basagoiti, A Belaustegi, I Bilbao, E Rodriguez, M Castaño. Hospital Universitario de Cruces, Pharmacy, Barakaldo, Spain

Background Linezolid is an antimicrobial approved for the treatment of hospital or community-acquired pneumonia and complicated skin and soft tissue infections due to Gram positive bacteria. Its use, though effective, is not free from possible harm.

Purpose To describe the incidence and nature of the adverse reactions related to linezolid, taking place before and after the 28-day limit given in the label information.

Materials and Methods All the linezolid treatments over one year (September 2011-September 2012) were recorded. Data sources were the electronic chart as well as the electronic prescription programme.

Results 280 cases were recorded, the median treatment duration being 8 days ( 1 to 73 days). 4 treatments were interrupted early due to potential interactions with antidepressants. A total of 27 patients developed adverse reactions.

Among the 255 patients treated for less than 28 days, 19 developed adverse reactions. 14 presented suppression of at least one myeloid cell line, 7 of them requiring transfusions (one with adverse skin reaction as well). Among the others, two had diarrhoea, one a skin reaction, one vomiting and the remaining patient, asthenia. Median treatment duration in patients with adverse reactions treated for less than 28 days was 12 days ( 3 to 27 days)

25 patients exceeded 28 days of treatment, 8 of whom had adverse reactions. Seven presented suppression of at least one myeloid cell line, 5 of whom required transfusion. The other patient suffered from asthenia. Median treatment duration in these patients was 37 days ( 32 to 56 days).

Conclusions Attention should be paid to blood cell counts from the beginning of the treatment, since, as seen, hematologic adverse reactions are not limited to treatments lasting more than 28 days. The same is applicable to other less frequent reactions such as skin reactions, vomiting and asthenia.

No conflict of interest.

\section{GRP-111 MANAGEMENT OF METHOTREXATE-INDUCED RENAL FAILURE WITHOUT GLUCARPIDASE}

doi:10.1136/ejhpharm-2013-000276.111

I Bilbao Meseguer, A De Basagoiti Gorordo, MA Gil Lemus, Z Baskaran Kaltzakorta, A Belaustegi Foronda, J Hernandez Goicoechea, B Baza Martinez, L Serrano De Lucas, A Bustinza Txertudi, B San Jose Ruiz. Hospital de Cruces, Pharmacy, Bilbao, Spain

Background Glucarpidase (Voraxaze) is effective in the treatment of methotrexate (MTX)-induced renal dysfunction but many cases this can be handled with standard treatment.

Purpose To describe the progress of a patient with MTX-induced renal failure in whose management glucarpidase was not used.
Materials and Methods A 13 year-old girl with acute lymphoblastic leukaemia treated with high-dose MTX. Baseline laboratory tests were normal, except for elevated transaminases and GGT.

Results The patient received her first consolidation cycle with $500 \mathrm{mg} / \mathrm{m}^{2}$ of MTX in 30 minutes, followed by $4500 \mathrm{mg} / \mathrm{m}^{2}$ in 23.5 hours, oral mercaptopurine $30 \mathrm{mg} / \mathrm{m}^{2} /$ day and triple intrathecal therapy. Simultaneously, she received hyperhydration/ alkalinisation (3000 $\mathrm{ml} / \mathrm{m}^{2} /$ day).There was no pharmacological interaction to MTX. 24 hours after the MTX infusion stared, the serum creatinine level ( $\mathrm{Cr}$ ) had tripled (see the table below). The following measures were taken: hyperhydration/alkalinisation $\left(4500 \mathrm{ml} / \mathrm{m}^{2} /\right.$ day $)$, colestyramine $(3 \mathrm{~g} / 6 \mathrm{~h})$ and folinic acid rescue at $500 \mathrm{mg} / \mathrm{m}^{2} / 6 \mathrm{~h} 31$ hours after the start of the MTX infusion. Although the protocol provides for the possibility of administering glucarpidase, it was decided not to do this because the methotrexate level was $<250 \mu \mathrm{M}$ and glucarpidase administration can be delayed until 96 hours after the start of MTX infusion. Difficulty in the subsequent monitoring, the absence of effect in renal function improvement and high cost were the reasons for delaying the treatment until at least having levels at 36 and 48 hours. Although $\mathrm{Cr}$ values were still high, elimination kinetics of the drug were seen as adequate. Without the use of glucarpidase, methotrexate levels were undetectable at day nine. The patient recovered her baseline renal function and did not have mucositis or liver toxicity.

Conclusions An early intervention with supportive treatment based on folinic acid, hyperhydration and urine alkalinisation was effective in the management of MTX-induced renal toxicity.

Abstract GRP-111 Table 1

\begin{tabular}{lll}
\hline $\begin{array}{l}\text { Time since MTX } \\
\text { infusion started }(\mathbf{h})\end{array}$ & $\mathbf{C r}(\mathbf{m g} / \mathbf{d L})$ & MTX levels $(\boldsymbol{\mu M})$ \\
\hline 0 & 0.35 & 0 \\
24 & 1.12 & 190 \\
36 & 1.41 & 24 \\
48 & 1.32 & 5.9 \\
\hline
\end{tabular}

No conflict of interest.

\section{GRP-112 MEDICAL DEVICES IN MOROCCO: WHAT GUARANTEES OF QUALITY AND SAFETY?}

doi:10.1136/ejhpharm-2013-000276.112

${ }^{1} \mathrm{MA}$ El Wartiti, 'W Enneffah, ${ }^{2} \mathrm{~A}$ Benmoussa, ${ }^{2} \mathrm{~J}$ Lamsaouri, ${ }^{3} \mathrm{~S}$ Makram, ${ }^{2} \mathrm{~J}$ Taoufik. ${ }^{1}$ Mohammed V Military Teaching Hospital - Faculty of Medicine and Pharmacy, Clinical Pharmacy - Therapeutic Chemistry, Rabat, Morocco; ${ }^{2}$ Faculty of Medicine and Pharmacy - Mohammed V University Souissi, Clinical Pharmacy - Therapeutic Chemistry, Rabat, Morocco; ${ }^{3}$ Mohammed V Military Teaching Hospital - Faculty of Medicine and Pharmacy, Pharmacy - Pharmacology, Rabat, Morocco

Background Nowadays, all over the world, many medical devices, initially considered as non-risk or low risk, have been proved to be extremely dangerous to human health, as evidenced by the latest scandal of PIP implants.

Purpose To report the experience of Mohammed V Military Teaching Hospital of Rabat in evaluating the quality and safety of medical devices and to analyse elements that can compromise the quality of these products in our country.

Materials and Methods 30-month prospective study (January 2010-June 2012). We collected claims relating to the quality of medical devices at our hospital, in normal conditions of acquisition, dispensing and use. We also analysed the processes of placing on the market medical devices, the systems governing their use in hospitals and the main Moroccan rules regulating them.

Results 30 claims were collected. They concerned: catheters (40\%), surgical drapes (20\%), gloves (17\%) and other medical devices $(23 \%)$. 